\title{
Laurence BINET
}

Médecins Sans Frontières International

\section{Médecins Sans Frontières en Tchétchénie de 1994 à 2004 : positionnements publics, questionnements et dilemmes}

Cet article a été rédigé à partir de l'étude « Crimes de guerre et politiques de terreur en Tchétchénie 1994-2004 » publiée dans la collection « Prises de parole publiques de Médecins Sans Frontières », par l'auteur de cette étude (Binet 2010). La collection, produite et publiée par Médecins Sans Frontières (MSF) a pour vocation initiale de mettre à disposition des volontaires un outil pédagogique leur permettant de s'approprier la culture de prise de parole publique de l'organisation humanitaire'. Ces études s'efforcent de décrire les processus de prise de décision qui ont mené aux positionnements publics de MSF lors de grandes crises humanitaires, ainsi que les dilemmes et controverses qui les ont accompagnés. Elles prennent la forme d'un récit chronologique composé d'extraits de documents propres à l'organisation (rapports de situation, de mission, échanges de courriels, comptes rendus de réunions, communiqués de presse, rapports de témoignages) et d'articles de presse. S'y ajoutent des extraits d'entretiens menés avec les protagonistes de MSF, acteurs des processus.

Les sources du présent article sont donc limitées à celles de l'étude sur la Tchétchénie. Les documents datent de l'époque des évènements, ce qui évite les tentations - volontaires ou non - de reconstruction de l'histoire a posteriori. Les

1 Longtemps réservées aux membres de l'association, ces études sont désormais mises à la disposition du public, sur le site $<$ http://www.speakingout.msf.org $>$. 
entretiens n'ont été menés qu'avec des membres de MSF. On peut toutefois trouver un regard et des éléments d'analyse extérieurs à l'organisation dans les articles de presse.

Après avoir décrit les positionnements publics de Médecins Sans Frontières concernant le conflit russo-tchétchène et dans quel contexte opérationnel ils s'inscrivaient, l'article se propose d'examiner les principaux questionnements et dilemmes que ces prises de paroles publiques ont posés à l'organisation aussi bien au cours du processus de décision des prises de paroles publiques qu'a posteriori.

\section{Ce que fait et ce que dit MSF}

Médecins Sans Frontières est une organisation humanitaire médicale de secours fondée par un groupe de médecins et de journalistes français en 1971. Au fil du temps, elle s'est transformée en un mouvement international de 23 entités (19 au moment des événements de Tchétchénie) composé de cinq centres opérationnels. Ces centres sont organisés autour des sections qui mènent effectivement des opérations, les sections belge, espagnole, française, hollandaise et suisse et des sections non opérationnelles qui leur apportent leur soutien en recrutant des ressources humaines, collectant des fonds, menant des actions de communication et de plaidoyer ${ }^{2}$. Les centres opérationnels ont une autonomie d'action. Mais les mécanismes de concertation se sont renforcés au cours du temps afin de limiter les incohérences et les risques opérationnels qui peuvent en découler. Les positionnements publics sont dans la mesure du possible portés au nom de Médecins Sans Frontières par l'ensemble du mouvement, après avoir fait l'objet de discussions et d'arbitrages internes parfois mouvementés.

MSF intervient pour la première fois dans l'espace soviétique lors du tremblement de terre en Arménie en 1988. En 1991 et 1992, l'organisation distribue des vivres et des médicaments dans les régions de Moscou et de Saint-Pétersbourg lors de la crise consécutive à la chute du régime soviétique. En 1993, elle ouvre une série de programmes : pour les sans-abris à Moscou, en Arménie et en Azerbaïdjan pendant la guerre du Karabagh, en Abkhazie et en Géorgie pendant le conflit abkhazo-géorgien. Par la suite, des programmes de soins aux patients tuberculeux sont mis en œuvre dans ces régions, ainsi que dans les prisons de Sibérie. En 1996, un programme VIH/Sida est ouvert à Moscou.

2 Les sections non opérationnelles sont toutefois de plus en plus intégrées dans les processus de discussion des stratégies opérationnelles. 
La première intervention de MSF dans le Caucase du Nord date de 1993 avec l'ouverture d'un programme auprès des réfugiés du Prigorodny en Ingouchie ${ }^{3}$, puis la prise en charge d'une épidémie de choléra en Tchétchénie à l'été 1994.

Le $1^{\text {er }}$ novembre 1991, cette république du Caucase du Nord s'est déclarée indépendante de la Fédération de Russie. En janvier 1992, le président tchétchène Djokhar Doudaïev a refusé de signer le traité d'adhésion de la Tchétchénie à la Fédération de Russie puis fait adopter une constitution tchétchène. En juin 1993, il dissout le parlement resté acquis à Moscou et s'attribue les pleins pouvoirs. Pendant l'été 1994, ses forces défont celles de l'opposition intérieure, soutenues et formées par Moscou.

En décembre 1994, les forces russes pénètrent sur le territoire tchétchène pour officiellement « désarmer les parties au conflit» (Cornell $2000: 210$ ). La guerre qui s'ensuit est présentée par les autorités russes comme une opération de police menée sur le territoire de la Fédération. Des villes et des villages entiers sont pilonnés et détruits par les forces fédérales qui entravent l'accès aux organisations humanitaires internationales (Le Parisien 1995 ; Cherkasov et Orlov 1998).

À partir de décembre 1994, les équipes de Médecins Sans Frontières s’efforcent de franchir les obstacles posés par les forces russes et de porter secours aux populations civiles sur le territoire tchétchène et dans les républiques voisines. Elles approvisionnent les hôpitaux en médicaments et matériel médical, opèrent les blessés, négocient et obtiennent parfois l'évacuation des patients lors des opérations de pilonnage des villages.

Communiqués et témoignages de volontaires dans la presse internationale décrivent la violence dont font preuve les troupes russes à l'égard des civils et les entraves qu'elles posent aux organisations de secours (Binet $2010: 26-27$ ).

En mai 1995, le bombardement massif de Chatoï et l'évacuation forcée de l'équipe MSF et de ses patients font l'objet d'une communication publique et d'un rapport dénonçant le non-respect du droit humanitaire par les forces russes (MSF 1995).

En avril 1996, après le pilonnage de plusieurs villes du sud de la Tchétchénie, renforcé par une interdiction d'accès aux organismes de secours, MSF tient une conférence de presse internationale à Moscou et diffuse un rapport documentant, à partir de récits de survivants et de volontaires, le ciblage des civils par les forces russes (MSF 1996). Quelques jours après cette conférence de presse l'une de ses

3 Fin 1992, un conflit armé éclate en Ossétie du Nord entre Ossètes et Ingouches à propos du district de Prigorodny. Ce district proche de la capitale, Vladikavkaz, qui avait été donné à l'Ossétie lors de la déportation des Ingouches en 1944, est revendiqué par ces derniers. Après les violences, des milliers d'Ingouches sont obligés de fuir l'Ossétie et de se réfugier en Ingouchie voisine. 
administratrices expatriées est prise en otage en Tchétchénie pendant plusieurs semaines.

À l'été 1996, le conflit s'achève par les accords de cessez-le-feu de Khassaviourt. L'armée russe se retire momentanément du pays. Le président Aslan Maskhadov, élu en 1997, ne parvient pas à ramener la stabilité dans un pays détruit par la guerre et gangréné par les mafias qu'elle a nourries et doit composer avec la montée des radicaux islamistes. Les enlèvements de cadres d'entreprises, travailleurs humanitaires et journalistes internationaux se multiplient, contribuant à déstabiliser le Caucase du Nord et à y décourager la présence d'étrangers (Sokirianskaya 2014).

Une succession de menaces, de cambriolages, d'attaques à main armée, de tentatives d'enlèvements, puis le meurtre de six employés du CICR en décembre 1996, amènent MSF à fermer progressivement ses programmes. En juillet 1997, l'administrateur de la section française est enlevé en Ingouchie. Il s'évade en octobre et dans la foulée le dernier programme de MSF dans le Caucase du Nord est clos.

En août 1999, un groupe armé dirigé par le chef de guerre et ancien premier ministre Chamil Bassaïev, qui agit sans en informer le président Maskhadov, et par Ibn El Khattab, un radical islamiste saoudien, mène une incursion armée au Daguestan. Plusieurs attentats à la bombe, qui font des centaines de victimes à Moscou, leur sont également attribués. Les forces russes interviennent alors de nouveau en Tchétchénie pour mener ce que Moscou qualifie cette fois d' «opération anti-terroriste ».

Dans ce contexte de guerre totale et d'insécurité majeure, les différentes sections de MSF éprouvent réticences et difficultés à mettre en œuvre des opérations au cœur du conflit et interviennent surtout auprès des réfugiés en Géorgie et en Ingouchie. Toutefois, malgré ce déploiement opérationnel limité, l'organisation dénonce publiquement la conduite de la guerre par les forces russes. En novembre, elle demande la réouverture immédiate de la frontière entre la Tchétchénie et la Géorgie et le respect du droit de fuite des civils tchétchènes (MSF 1999b). En décembre, en recevant le prix Nobel de la Paix à Oslo, ses représentants lancent un appel au cessez-le-feu à Grozny, la capitale tchétchène, dont la population civile est menacée d'être considérée comme terroriste et exterminée (Le Huérou et Regamey 2012). Début 2000, MSF diffuse à la presse internationale un rapport composé de récits de réfugiés tchétchènes en Géorgie et qualifie de « guerre » ce que les autorités russes continuent à présenter comme une opération anti-terroriste (MSF 1999c).

Au printemps 2000, après une période de bombardements intensifs qui anéantit villes et villages et pousse plus de 200000 Tchétchènes à se réfugier dans les républiques voisines, s'instaure une phase qualifiée de «normalisation» par les autorités fédérales. Elle se caractérise par l'imposition de la terreur qui fait disparaître des milliers de civils dans les opérations de nettoyage et les camps de torture de l'armée russe (Gilligan 2009). De son côté, une partie de la résistance tchétchène se 
radicalise, débordant le président Maskhadov, dont la légitimité n'est plus reconnue par Moscou. Les groupes armés multiplient les attentats contre les représentants du pouvoir fédéral et l'administration tchétchène pro-russe que le Kremlin met en place.

Les différents centres opérationnels de MSF lancent des activités de soutien aux réfugiés dans les républiques voisines de Géorgie, d'Ingouchie et du Daguestan. En Tchétchénie, les opérations d'approvisionnement des hôpitaux en médicaments et matériel médical sont réalisées par du personnel local, formé et supervisé à distance par des équipes d'expatriés basées à Moscou et à Nazran, qui se rendent ponctuellement sur le terrain.

$\mathrm{Au}$ cours de leurs activités dans les structures de santé tchétchènes, les équipes recueillent des récits de patients victimes de violences, qui dressent le portrait d'une population vivant sous la terreur. À l'automne 2000, ces récits servent de base à une campagne de communication en Europe, portée aussi bien dans les médias d'Europe de l'Ouest et d'Amérique du Nord que dans les médias russes par un des chefs de mission de MSF (Mac Mahon 2000) ${ }^{4}$. En janvier 2001, ce dernier est pris en otage pendant trois semaines.

En Ingouchie, les efforts des équipes de MSF pour améliorer concrètement les conditions de vie des réfugiés se heurtent à la volonté des autorités ingouches et fédérales de maintenir ces derniers dans la précarité pour les pousser à rentrer en Tchétchénie. Ce retour doit convaincre les États et les institutions internationales que la situation y est en voie de normalisation. En réalité, les conditions de vie et de sécurité dans ce pays en ruine sont catastrophiques. Les secours qui parviennent à la population tchétchène sont dérisoires en raison du climat de terreur, des actions de harcèlement administratif, voire de détournements entrepris par des forces armées et une administration corrompues qui compromettent les actions des organisations de secours (MSF 2002d).

En janvier 2002, MSF lance une campagne de communication dénonçant ces pressions au rapatriement (MSF 2002c). Le temps d'une conférence de presse à Paris, elle s'associe à Sergueï Kovalev, ancien dissident, député de la Douma russe et militant des droits de l'homme (AFP 2002). Cette campagne se prolonge au printemps et à l'été 2002, contre le plan de rapatriement en 20 étapes établi par les autorités russes et ingouches qui formalise le démantèlement par la force des camps de réfugiés tchétchènes et le retour de ceux-ci dans leur pays dévasté (MSF 2002g).

À l'été 2002, alertes, menaces, tentatives et incidents d'enlèvement se multiplient dans le Caucase du Nord. Le 12 août 2002, le chef de mission de la section suisse de MSF au Daguestan, de nationalité néerlandaise, est enlevé à Makhachkala, la capitale

4 Voir également l'interview de Colin Mac Mahon dans le programme « Glas Naroda » de la chaîne de télévision russe NTV le 22 décembre 2000. 
de la république. Certains éléments indiquant rapidement que l'enlèvement ne relève pas du pur banditisme, la peur qu'une prise de parole trop accusatrice ferme toutes les pistes incite les responsables à la prudence. Pendant les six mois qui suivent, la communication de l'organisation concernant cet événement se limite donc à demander la libération de son volontaire.

En octobre 2002, MSF est sollicitée par les autorités russes pour jouer le rôle de médiateur à la demande des rebelles qui ont pris en otage 800 personnes au théâtre de la Doubrovka à Moscou. Le responsable de MSF sur place refuse, arguant que la négociation ne relève pas du mandat de l'organisation. Il propose toutefois un soutien médical si nécessaire. Finalement, c'est le CICR qui négocie. Après l'assaut aux gaz lancé par les forces russes sur le théâtre, une équipe de MSF apporte son soutien à l'un des hôpitaux accueillant les centaines de personnes intoxiquées. Elle y est le témoin de la situation sanitaire désastreuse qui règne dans l'hôpital en raison du manque de moyens et de préparation du personnel à la prise en charge de ce type de pathologie, le tout aggravé par le manque d'informations fournies par les autorités sur la nature du gaz utilisé. Si les responsables de MSF expliquent publiquement le refus de mener la négociation, ils restent silencieux sur la situation dont les équipes ont été témoins au sein de l'hôpital (MSF 2002h).

À partir de janvier 2003, toujours sans nouvelles de leur chef de mission enlevé au Daguestan, ils décident de changer de stratégie et de donner plus de visibilité médiatique à l'enlèvement : lancement d'une pétition mondiale demandant sa libération ; communiqués de presse marquant les dates-anniversaires ou l'obtention de preuves de vie ; conférences de presse avec la famille et des représentants des autorités néerlandaises, etc. (MSF 2003a).

Dans ses communiqués et ses déclarations à la presse, l'organisation met en avant le manque d'implication des autorités russes dans l'enquête et le peu d'efforts fournis pour obtenir la libération de l'otage, alors que ce dernier a été enlevé sur leur territoire. À partir de mars 2004, sur la base d'éléments obtenus auprès d'enquêteurs privés et de journalistes indépendants, une stratégie plus offensive est lancée qui accuse des membres des parlements russe et daguestanais et pointe l'implication des services spéciaux russes.

Parallèlement, l'organisation continue à dénoncer publiquement les pressions au rapatriement des réfugiés en Tchétchénie. En mars 2003, une lettre est envoyée à Mourat Ziazikov, le président ingouche, lui demandant de laisser les réfugiés tchétchènes s'installer dans des logements construits par l'équipe de MSF avec l'accord de l'administration locale (MSF 2003b). Cette lettre est envoyée à divers responsables occidentaux afin qu'ils appuient la demande. En mai, à l'occasion d'une conférence de presse à Moscou, MSF rend publique une enquête menée auprès des 
réfugiés, qui montre que plus de $90 \%$ d'entre eux refusent de rentrer en Tchétchénie, essentiellement pour des raisons de sécurité (MSF 2003c).

Pendant toute cette période de crise, MSF accompagne toutes ses prises de parole publiques sur la situation en Tchétchénie, le sort des réfugiés et l'enlèvement de ses volontaires, de rencontres et de pressions diplomatiques. Ainsi, toutes les occasions de visites d'officiels russes dans des États européens ou nord-américains et de sommets internationaux incluant la Fédération de Russie sont saisies pour interpeller publiquement les uns et les autres. À trois reprises, en janvier et en novembre 2000 puis en janvier 2002 (MSF 2002a), MSF est auditionnée par le Conseil de l'Europe sur la situation en Tchétchénie et sur le sort des réfugiés. En avril 2002, ses représentants sont entendus par la Commission des droits de l'homme de l'ONU (MSF 2002e). Chacune de ces auditions fait l'objet d'une communication publique.

MSF demande aux autorités russes de cesser les rapatriements forcés des réfugiés dans la république de Tchétchénie où la «normalisation » s'opère dans la terreur et la misère. L'organisation rappelle aussi aux pouvoirs russes qu'il est de leur responsabilité de faire libérer le chef de mission enlevé au Daguestan, donc sur le territoire de la Fédération. Enfin, elle en appelle aux chefs d'États étrangers et aux organisations internationales afin qu'ils fassent tout pour convaincre leurs interlocuteurs russes de résoudre ces problèmes.

L'otage est finalement libéré le 11 avril 2004. Pendant les dix-huit mois qu'a duré l'enlèvement, le personnel national de MSF en Ingouchie et en Tchétchénie a continué à mener des opérations de secours dans ces deux républiques. Le personnel international ne reviendra que plusieurs années plus tard, progressivement et pour de courts séjours. Une équipe d'expatriés se réinstallera en Tchétchénie de façon permanente en 2011.

\section{Dilemmes et questionnements}

Tout au long de cette période de crise, les positionnements opérationnels et l'expression de MSF dans l'espace public suscitent questionnements et dilemmes et donnent lieu à débats et controverses tant au sein de l'organisation qu'avec ses interlocuteurs extérieurs.

\subsection{Faut-il ignorer les accusations d'espionnage régulièrement émises contre MSF dans les médias russes, ou y répondre?}

En février et mars 2000, MSF est confronté à une série de déclarations publiques dans les médias de la Fédération de Russie qui mettent en cause la neutralité de sa présence dans le Caucase. 
Le 29 février, le ministre de la justice russe déclare publiquement que « les forces russes ne mettent pas d'obstacle au transport par la Croix-Rouge et Médecins Sans Frontières de médicaments destinés aux combattants indépendantistes tchétchènes dans les zones montagneuses » (MSF 2000b). Ces propos diffusés par les agences de presse sont rapportés par la chaîne russe Ren TV sous la forme d'une accusation. Invitées à venir expliquer à l'écran « pourquoi MSF livre des médicaments aux indépendantistes », les équipes de MSF déclinent l'interview en expliquant qu'elles ne livrent pas de médicaments aux indépendantistes (MSF 2000b).

Le 4 mars, le commandant des forces russes en Tchétchénie déclare à la télévision russe que « MSF a des intérêts qui portent préjudice à l'État russe » (MSF 2000c ; Binet 2010 : 110-111). Au sein de l'organisation, certains, dont ceux qui gèrent d'autres projets en Fédération de Russie, traiteraient volontiers ces déclarations par l'indifférence, soulignant que toutes les organisations d'origine étrangère en Russie font régulièrement l'objet de ce genre de provocation. Une simple réaction de principe afin de ne pas hypothéquer les autres projets d'intervention de l'organisation dans la région leur semble suffisante. Mais d'autres, moins impliqués en Russie hors du Caucase, considèrent cet incident comme majeur et souhaitent une réaction forte. Une lettre est donc envoyée par la chargée des relations de MSF avec les Nations Unies à l'ambassadeur de la Fédération de Russie aux Nations Unies. La lettre qualifie les déclarations du commandant des forces russes de «calomnie, pouvant mettre en danger les possibilités d'action des organisations de secours international dans le Caucase » (MSF 2000c).

Une semaine plus tard, l'agence de presse officielle russe Itar Tass affirme que le bureau de MSF dans la vallée de la Pankissi en Géorgie a été ouvert « pour servir aux trafics de matériel humanitaire et d'armes des combattants tchétchènes » (Binet 2010 : 112). Comme partout ailleurs, les équipes de MSF y soignent effectivement tous les blessés qui leur sont amenés, quelle que soit leur origine. Parmi eux se trouvent des combattants blessés, pour lesquels d'après les Conventions de Genève « la qualité de malade prime sur celle de combattant aussi longtemps que la blessure ou la maladie le met hors d'état de combattre » (Bouchet-Saulnier 2013 : 75). Au final les responsables de MSF interprètent ces accusations comme les éléments d'une stratégie destinée à discréditer la Géorgie, montrée du doigt pour accueillir des « terroristes », plutôt que MSF. Ils décident de ne pas y répondre publiquement.

Cette série d'incidents incite toutefois MSF à développer des relations plus suivies avec les médias de la Fédération de Russie afin que ses activités y soient mieux et plus souvent décrites. 
2.2 Dans un contexte de terreur, face à un régime qui nie la réalité de la guerre, en quoi est-il utile que cette situation soit qualifiée de "guerre "? Est-ce le rôle de MSF de plaider pour cette qualification?

Lors de la «première guerre », les prises de paroles publiques de MSF dénoncent le non respect du droit international humanitaire par les troupes de la Fédération de Russie qui bombardent de façon indiscriminée les populations civiles et les structures médicales et entravent l'accès des organisations de secours (MSF 1995). Ces messages s'adressent aux autorités de la Fédération de Russie, mais aussi à la communauté internationale, dans l'espoir que celle-ci puisse contribuer à imposer aux troupes russes le respect du droit humanitaire international (DIH) dans la conduite de la guerre.

De son côté, Moscou affirme mener une opération de « restauration de l'ordre » dans l'une des républiques du territoire de la Fédération. Il n'est pas question de guerre, donc pas question de droit humanitaire international à appliquer. Lorsque les hostilités reprennent en 1999, elles sont qualifiées « d'opération anti-terroriste ».

MSF se positionne alors publiquement pour que cette situation soit qualifiée de « guerre » par les États du Conseil de l'Europe ce qui obligerait la Russie à respecter les règles du droit humanitaire vis-à-vis des populations (MSF 2000a). Au sein de l'organisation, deux positions se dessinent. Les uns questionnent cette démarche qui selon eux risque d'être comprise comme une participation au débat politique sur la légitimité des revendications indépendantistes tchétchènes. La neutralité de MSF pourrait ainsi être mise en question, alors qu'elle est la garantie indispensable pour avoir accès à toutes les populations en danger. Il serait donc préférable de demander que toutes les parties au conflit respectent les Conventions de Genève. Les autres avancent que personne ne conteste la légitimité des dites conventions dans cette situation et soulignent que le problème relève plutôt du fait que les États ne condamnent pas suffisamment la Fédération de Russie pour les violer.

Le 27 janvier 2000, le ministre des Affaires étrangères russe tranche la question en martelant devant le Conseil de l'Europe que les événements de Tchétchénie ne sont pas « un conflit», ni une « guerre », mais une « action anti-terroriste » et que « l'usage de la force par l'armée russe est proportionné à la situation »(AFP 2000)5.

5 La position de la Russie n'évoluera pas jusqu'à la proclamation de la fin de l'opération anti-terroriste en 2009. 
2.3 Malgré les risques d'agressions et d'enlèvements encourus par le personnel dans le Caucase du Nord, peut-on quand même établir une présence opérationnelle a minima en la justifiant par la nécessité d'alimenter une prise de parole publique qui dénonce les persécutions subies par la population tchétchène?

La question se pose à nouveau aux responsables de MSF à la reprise de la guerre en 1999. Comment porter secours aux milliers de civils tchétchènes qui subissent ou fuient les bombardements, mais aussi convaincre qu'assistance et protection doivent impérativement leur être apportées ? Les responsables de MSF ont en mémoire les graves incidents de sécurité et les multiples entraves posées à son action dans le contexte de guerre totale du premier conflit. Leurs réticences à envoyer du personnel dans ce qui est perçu comme un enfer, sans garantie qu'il puisse agir, n'ont d'égal que le sentiment de culpabilité de ne pas intervenir. Ce dilemme est exposé dans les médias (Bohlen 1999). "Pourquoi nous n’y sommes pas ? Pardon de ne pas y être » résume, non sans ironie, un responsable qui critique le «nombrilisme humanitaire » exposé dans les médias et essaie de convaincre ses collègues d'aller de l'avant (MSF 1999a).

Des opérations d'assistance sont finalement mises en œuvre auprès des réfugiés en Ingouchie et en Géorgie, puis, au bout de quelques mois, des distributions de médicaments et de matériel médical sont organisées en Tchétchénie, par le personnel local. Et c'est à partir de cette légitimité opérationnelle que MSF dénonce publiquement la conduite de la guerre, en publiant des récits recueillis auprès des réfugiés et, plus tard, auprès des patients des structures de santé tchétchènes. En effet, MSF n'étant pas une organisation de défense des droits de l'homme, mais une organisation de secours médical, elle ne s'autorise que des prises de parole basées sur les constats réalisés par ces équipes dans le cadre de leurs activités opérationnelles. Une exception : lorsqu'aucun autre témoin ne peut dénoncer les crimes dont l'organisation a connaissance. Il s'agit de conserver une plus-value à cette parole, de ne pas l'affaiblir en la rendant systématique ou en la transformant en simple écho de celle d'autres témoins.

\subsection{Les prises de parole publiques sur le Caucase du Nord doivent-elles être modérées pour ne pas compromettre les activités dans les autres régions de la Fédération de Russie?}

La question se pose essentiellement au centre opérationnel de MSF qui gère également un programme pour les sans-abris à Moscou et un programme anti-tuberculose en Sibérie. Ses responsables craignent qu'un positionnement trop vocal sur le Caucase $\mathrm{du}$ Nord nuise aux négociations en cours pour les autres programmes. Après avoir essayé en vain pendant quelques temps de tenir leur centre opérationnel à distance des prises de parole publiques du reste du mouvement, les responsables décident de mettre en place deux équipes de coordination autonomes : l'une pour le Caucase, l'autre pour 
les programmes dans d'autres régions de la Fédération de Russie. Ils veulent croire que cette stratégie de « cloisonnement » permettra de distinguer les positionnements publics des différents programmes sans confondre les enjeux.

Et c'est officiellement en raison d'un désaccord avec les autorités sanitaires russes sur les protocoles médicaux que le programme anti-tuberculose fermera ses portes en septembre 2003 (MSF 2003d). Rien ne laisse croire que les prises de parole publiques de MSF sur le Caucase ont joué un rôle dans cette fermeture.

2.5 Existe-t-il - comme la chronologie des évènements pourrait le laisser penser - un lien de cause à effet entre les prises de parole publiques de MSF et les incidents de sécurité contre son personnel? Faut-il tenir compte de cette éventualité dans les décisions de prise de parole publique, et comment?

La tentation est grande de voir derrière chaque enlèvement les cibles des prises de parole dénonciatrices qui l'ont précédé. Ces enlèvements viseraient à obtenir le silence de MSF, voire la fermeture de ses programmes, l'organisation espérant ainsi protéger la vie de l'otage et éviter de nouveaux incidents de sécurité. Cette lecture est pourtant loin d'être systématiquement confirmée par les éléments d'information recueillis sur l'identité des ravisseurs. Mais elle reste la plus spontanée et la plus courante, en particulier au sein du centre opérationnel dont l'administratrice a été enlevée en 1996, quelques semaines après la tenue d'une conférence de presse à Moscou. Nombreux sont ceux qui restent persuadés que cette prise d'otage était liée à l'expression publique de l'organisation. Pendant la première année du second conflit, hantés par ce risque, ils plaident pour une limitation des prises de position publiques de MSF concernant la Tchétchénie. D'autres ne se résignent pas à se taire, mais préfèreraient toutefois que MSF « témoigne discrètement » (Binet 2010 : 255-256). Cet oxymore révèle un conflit entre leur conscience qui les pousse à se prononcer en faveur de la dénonciation, et leurs craintes face aux risques d'enlèvement qu'ils pensent, sans doute faute d'autre explication répondant à une logique lisible, renforcés par la prise de parole publique.

La diffusion en août 2000, dans les médias russes et internationaux, des résultats d'une enquête menée par le personnel national montrant que $70 \%$ des réfugiés tchétchènes en Ingouchie ne souhaitent pas rentrer dans leur pays pour des raisons de sécurité (MSF 2000d) donne l'occasion d'une nouvelle manifestation de cette approche ambiguë. En effet, elle s'effectue sous la forme peu attractive d'une distribution aux médias de "fiches d'information » non signées par MSF, au lieu d'un classique communiqué de presse assumé et signé par l'association et portant son positionnement. Cette absence de signature d'une information pourtant significative limite effectivement les risques inhérents à cette communication, mais elle en en limite surtout l'impact. 
D'autres responsables de MSF qui connaissent bien la région se montrent plus circonspects et prudents à lier systématiquement prises de parole publiques et enlèvements. D'une part, ils rappellent que ce sont les habitants du Caucase qui sont les premières victimes des kidnappings. D'autre part, ils soulignent que le fait que les otages rapportent avoir été gardés, voire enlevés par des Caucasiens et non par des Russes ne dit rien sur les commanditaires de leur enlèvement. À l'inverse, l'existence de demandes de rançons ne suffit pas à ranger un kidnapping dans la catégorie « mafieux-criminel ». Les objectifs peuvent être tout à la fois financiers et politiques - en fait ils le sont presque toujours dans ce contexte de terreur et de misère, la rançon servant à gagner de l'argent et à « payer les frais » de l'opération, mais aussi à donner une apparence mafieuse à un crime aux objectifs politiques ${ }^{6}$.

En janvier 2001, l'enlèvement du chef de mission qui vient de porter à haute voix une campagne de dénonciation de MSF semble désigner comme responsables les services de la Fédération de Russie, ciblée par cette campagne. Or, quelques heures avant sa libération, l'otage se voit remettre une lettre de Chamil Bassaïev, l'un des chefs indépendantistes tchétchènes. Ce dernier s'y excuse de cet enlèvement qu'il présente comme un «malentendu »: « un groupe de nos Moudjahidines a décidé de son propre chef d'obtenir la libération de plusieurs de nos camarades en échange de votre personne. » (Bassaïev 2001)

Lorsqu'à partir de l'été 2002, les enlèvements se multiplient dans le Caucase - dont celui du chef de mission de MSF au Daguestan - la presse internationale rapporte que la plupart des organisations internationales, qui s'expriment sous couvert d'anonymat, y voient autant de pressions pour les faire partir du Caucase du Nord et obliger les déplacés, ainsi privés d'aide humanitaire, à rentrer en Tchétchénie (Talbi 2002).

$\mathrm{Au}$ final, au-delà de toute spéculation sur l'identité de ceux « à qui profiteraient le crime ", l'idée que se positionner publiquement, c'est prendre obligatoirement des risques pour la sécurité des équipes et des programmes reste omniprésente dans les réflexions qui préparent aux prises de parole publiques de MSF, quelle que soit la région du monde concernée. Mais elle est particulièrement aigue à cette époque et imprègne toute la réflexion sur les actions de MSF en Fédération de Russie.

6 Discussions internes à MSF rapportées à l'auteur. On trouvera des traces écrites de discussions semblables en 1997 et 2001 dans les procès-verbaux du conseil d'administration de MSF in Binet 2010 : 66-67, 150-151. 
2.6 Lorsqu'un membre du personnel est pris en otage, faut-il s'exprimer dans les médias pour hii donner une visibilité qui le protégerait ou au contraire rester le plus discret possible pour éviter d'accroître sa "valeur marchande "?

À cette question qui concerne au demeurant toutes les situations de prises d'otage dans le monde, MSF répond au cas par cas en fonction de sa compréhension du contexte de chacun des enlèvements.

Lors des prises d'otage de la première guerre russo-tchétchène et de l'entre-deuxguerres, c'est la stratégie de la discrétion qui est adoptée, soit parce qu'on pense que c'est un positionnement public qui a suscité le kidnapping et qu'il faut éviter de jeter de l'huile sur le feu (c'est le cas lors de l'enlèvement de l'administratrice en 1996), soit parce qu'on pense qu'une prise de parole publique n'est d'aucune utilité dans la résolution d'un enlèvement qu'on soupçonne être de nature purement criminelle, voire qu'elle risque de donner une « valeur marchande » à l'otage. C'est le cas lors de l'enlèvement de l'administrateur en Ingouchie en 1997 au cours duquel MSF choisit de ne communiquer qu'avec les rares journalistes susceptibles de lui apporter des informations. De même, par souci d'indépendance, les relations avec le gouvernement français sont courtoises, mais réduites au minimum que requiert la nationalité française de l'otage.

En revanche, en janvier 2001, MSF s'exprime sur l'enlèvement de son chef de mission (New York Times 2001) en particulier dans les médias de la Fédération de Russie (MSF 2001). En effet, l'organisation soupçonne un acte de représailles après la forte implication de ce dernier dans une campagne de dénonciation, de sorte qu'une forme de pression publique et politique semble aller de soi.

2.7 Faut-il pointer du doigt publiquement les responsabilités des autorités du territoire sur lequel l'otage a été enlevé, voire leur négligence etéventuellement leur complicité, afin de les obliger à agir pour sa libération, ou bien s'en abstenir pour éviter d'obtenir l'effet inverse en les braquant?

Ces questions se posent de manière particulièrement aiguë lorsque plusieurs mois après l'enlèvement, en août 2002, du chef de mission au Daguestan, les responsables de MSF s'aperçoivent que les autorités de police locales et fédérales ont mis fin aux recherches depuis des semaines, sans même prévenir la famille, ni l'organisation.

Le mouvement MSF choisit alors de briser le silence et de passer à une stratégie de responsabilisation des autorités sur le territoire desquelles s'est déroulé l'enlèvement - soit celles du Daguestan et de la Fédération de Russie - et des États et organismes internationaux susceptibles de les convaincre de s'activer pour obtenir la libération de l'otage. Cette stratégie d'interpellation publique monte en puissance au fur et à mesure que les éléments des enquêtes menées en parallèle par un journaliste russe et son confrère néerlandais orientent les soupçons vers des individus proches 
d'hommes d'affaires et de responsables politiques russes et daguestanais (Van Zwol 2003a, 2003b ; Izmaïlov 2003, 2004). Toutefois, l'objectif n'est pas tant de désigner les coupables que de faire pression pour que ceux qui ont de l'influence sur eux les poussent à relâcher l'otage.

Le dilemme du lien direct entre prise de parole publique et sécurité refait alors surface : la mise en lumière de ces éventuels responsables du kidnapping ne risquet-elle pas de nuire à l'otage ? C'est la crainte de certains responsables de la section néerlandaise de MSF, qui soutiennent la position de réserve très diplomatique des autorités des Pays-Bas et de la famille de l'otage : maintenir un profil bas afin de ne pas braquer les autorités russes, acteur clé dans un éventuel processus de libération.

En fait, la section néerlandaise est confrontée au dilemme suivant : peut-elle se positionner à contre-courant de l'opinion publique des Pays-Bas - donc de ses soutiens et donateurs potentiels - qui suit la ligne de prudence diplomatique de son gouvernement? Il est renforcé par le fait que la majorité des responsables de son exécutif ne sont pas néerlandophones et peinent à expliquer la situation à une société néerlandaise qu'ils connaissent mal.

La position de la section suisse, employeur officiel d'Arkan Erkel, est plus proactive et bénéficie du soutien du reste du mouvement MSF pour mener une stratégie de pression publique. Celle-ci est mise en œuvre (MSF 2004), au prix de lourdes tensions qui pèseront un temps sur la cohésion du mouvement.

\subsection{Faut-il continuer à dénoncer publiquement les violences faites aux populations dans la région, au risque de radicaliser les parties au conflit qui seraient commanditaires de l'enlevement et mettre la vie de l'otage en danger?}

Lors de la prise d'otage du théâtre de la Doubrovka en octobre 2002, certains responsables de MSF craignent qu'un rôle d'intermédiaire dans une négociation ne compromette une éventuelle ouverture dans la recherche du chef de mission enlevé au Daguestan. D'autres, au contraire, pensent qu'il est du devoir d'une organisation humanitaire de faire tout ce qu'elle peut pour aider des otages. Ils voient aussi une opportunité de prise de contacts avec les autorités russes qui n'ont jusqu'ici accordé aucun rendez-vous à haut niveau aux responsables de l'organisation pour parler de l'enlèvement.

Le dilemme est tranché de facto quand le CICR prend en charge la médiation et que la presse russe révèle que les preneurs d'otage avaient demandé la médiation de MSF. L'organisation justifie alors sa position dans un communiqué (MSF 2002f) et des interviews à la presse (Gnabé 2002) qui précisent les conditions de sa présence sur les lieux du drame : répondre aux éventuels besoins médicaux et humanitaires.

Quant à son silence sur la situation dans l'hôpital qui accueille les spectateurs intoxiqués au gaz, il est justifié en interne par le souci de ne pas nuire à l'otage, 
mais aussi au personnel de l'hôpital, en particulier à ceux qui eux-mêmes intoxiqués en donnant des soins se sont vus recommander la plus grande discrétion par les « services » russes. Enfin les médias occidentaux couvriraient suffisamment bien le sujet pour que MSF n'ait pas besoin d'intervenir à son tour.

Six mois plus tard, alors que l'organisation vient d'opter pour une stratégie d'interpellation publique concernant l'enlèvement de son chef de mission, deux de ses responsables basés à Moscou racontent à un quotidien néerlandais ce qu'a vu l'équipe MSF dans l'hôpital après les évènements de la Doubrovka et pourquoi elle n'en a pas fait état plus tôt (Veldkamp et Van Zwol, 2003).

Pendant toute cette période, comme si de toute façon le pire avait été atteint avec l'enlèvement du Daguestan, MSF dénonce sans relâche la pression au rapatriement forcé imposée aux Tchétchènes des camps d'Ingouchie. Publications de rapports et conférences de presse se succèdent malgré l'atmosphère de terreur qui touche les populations et de plus en plus les humanitaires. Les chefs de mission de MSF à Moscou font l'objet de menaces et d'actes d'intimidation : cambriolage des appartements, passage à tabac par des inconnus d'un des coordinateurs expatriés et refus temporaire des autorités russes de laisser sa famille, britannique, sortir du territoire, etc. Cette escalade prend fin lorsque tous les camps d'Ingouchie sont démantelés et la majorité des réfugiés de retour en Tchétchénie (Binet 2010 : 254-255).

\section{Conclusion}

Pendant toutes ces années, tous les positionnements publics de Médecins Sans Frontières s'élaborent à l'ombre d'un questionnement récurrent qui est propre au contexte de la Fédération de Russie : la prise de parole publique via l'interpellation des États-membres de l'ONU et la mobilisation de leurs opinions publiques est-elle un moyen d'action pertinent face à la Russie, super-puissance possédant un veto au Conseil de sécurité de l'ONU et une tradition de contrôle propagandiste de l'espace public, héritière d'un passé qui ignorait largement la liberté d'expression, mais aussi premier fournisseur de gaz des États européens ? Peu d'autres contextes - à l'exception de la Chine, que MSF n'a pas eu l'occasion d'interpeller publiquement - présentent un tel éventail de contraintes lorsqu'il s'agit de s'exprimer dans l'espace public pour débloquer une situation.

En tant qu'organisation humanitaire, MSF doit tenter tout ce qui est possible pour que les équipes puissent être en mesure de porter secours aux populations en détresse, sans toutefois se faire aucune illusion sur le fait que ce « possible » puisse se matérialiser et permettre réellement d'améliorer le sort des populations.

Dans un premier temps, la campagne de sensibilisation menée tous azimuts par MSF et d'autres organisations humanitaires ou de défense des droits de l'Homme auprès des États et des Institutions internationales connaît quelques succès modestes 
et ponctuels. Ainsi en avril 2000, l'Assemblée parlementaire du Conseil de l'Europe vote unanimement en faveur de la suspension de la Russie jusqu'à ce que cette dernière commence à rechercher la paix et à mettre fin aux violations des droits de l'homme en Tchétchénie (APCE 2000). La suspension est levée neuf mois plus tard, sans qu'aucun progrès n'ait été constaté. Au fil du temps, l'APCE se montrera de plus en plus tolérante face aux agissements de la Russie et prônera même le retour des déplacés en Tchétchénie.

Après les attentats du 11 septembre 2001, si la mobilisation des opinions publiques reste importante, elle ne suffit plus à convaincre les responsables politiques de s'opposer à leurs homologues de la Fédération de Russie. Ces derniers se drapent dans la rhétorique de la lutte anti-terroriste qui s'impose désormais dans les relations internationales, annihilant ainsi toute velléité de réaction de la part des États comme des institutions internationales. MSF continue toutefois à les interpeller, mais c'est désormais pour les accuser de « collaboration » (MSF 2002b) pour demander « quelle instance internationale est aujourd'hui en mesure de faire cesser en Tchétchénie les violations massives des droits de l'homme et du droit international » (MSF 2002e) ou pour qualifier de « cosmétiques » leurs recommandations pour alléger le sort des Tchétchènes (MSF 2002f). L'organisation ne se fait plus aucune illusion sur l'impact de ses positionnements qu'elle continue toutefois à porter publiquement par souci de responsabilité.

Un responsable de Médecins Sans Frontières résume ainsi cette approche :

On espérait toujours qu'en communiquant et en faisant un travail de plaidoyer, on allait influencer un certain nombre de choses au niveau de la violence, au niveau du respect du droit international humanitaire, au niveau du comportement des chancelleries et de la communauté internationale, au niveau des opérations, en défendant un espace de travail sur place. Mais on le faisait par souci de responsabilité. On savait très bien que compte-tenu du poids de la Russie, rien ne changerait, que la Russie avait un blancseing pour mater la rébellion tchétchène et pour passer à la trappe une bonne partie du peuple tchétchène. Mais pour autant, on n'a pas laissé tomber. Malgré la conscience très nette qu'on n'arriverait pas à faire changer tout cela, on estimait qu'on avait la responsabilité d'essayer (Binet $2010: 351$ ).

C'est cette « éthique de responsabilité » qu'ont en tête les représentants de MSF lorsqu'à la remise du prix Nobel de la paix à Oslo le 10 décembre 1999, ils appellent à l'arrêt des bombardements de la capitale tchétchène (MSF 1999d) et manifestent devant l'ambassade de Russie vêtus de T-shirts portant l'inscription « Grozny » en lettres rouge sang.

«Quand on reçoit le prix Nobel la veille de l'expiration d'un ultimatum, on ne peut pas, devant l'ambassadeur de Russie, décemment faire autre chose. On se serait déshonorés, à parler la langue de bois fleurie au cours d'une cérémonie comme cela. Mais c'est tout » (Binet 2010 : 93), résumera quelques années plus tard le docteur Rony Brauman, ancien président de MSF, présent à la cérémonie. 


\section{Bibliographie}

AFP 2000 : « Doctors without Borders demand Action on Chechnya », Dépêche AFP, Strasbourg (Conseil de l'Europe), 27.01.2000 (in Binet 2010).

AFP 2002 : « Le député russe Kovalev dénonce la frilosité de l'Occident sur la Tchétchénie », Dépêche AFP, 25.01.2002.

APCE 2000 : «Conflit en République tchétchène. Mise en œuvre par la Fédération de Russie de la Recommandation 1444 (2000) », Recommandation 1456 de l'Assemblée Parlementaire du Conseil de l'Europe, 06.04.2000, document en ligne, consulté le 13.10.2011 <http://assembly.coe.int/Mainf.asp?link=/Documents/AdoptedText/ta00/ FREC1456.htm>.

Bassaïev 2001 : Lettre de Chamil Bassaïev à Kenneth Gluck [chef de mission MSF], version postée sur le site kavkazcenter.net le 12.03.2001 (in Binet 2010).

Binet 2010 : Laurence Binet, Crimes de guerre et politiques de terreur en Tchétchénie 19942004, Médecins sans frontières, "Prises de parole publiques de Médecins Sans Frontières », 2014, document en ligne, consulté le 14.11.2014 <http://speakingout.msf. org/fr/crimes-de-guerre-et-politiques-de-terreur-en-tchetchenie>.

Bohlen 1999 : Celestine Bohlen, «Fears Rise for Chechen Refugees as Winter Nears », The New York Times, 30.10.1999.

Bouchet-Saulnier 2013 : Françoise Bouchet-Saulnier, Dictionnaire pratique du droit humanitaire, Paris : La Découverte, 2013.

Cherkasov et Orlov 1998 : Alexandre Cherkasov et Oleg Orlov, Russia-Chechnya: Chain of Mistakes and Crimes, Moscou, 1998, document en ligne, consulté le 05.05.2013 $<\mathrm{http}: / / \mathrm{memo} . \mathrm{ru} / \mathrm{hr} /$ hotpoints/chechen/checheng/>.

Cornell 2000 : Svante Cornell, Small Nations and Great Powers: A Study of Ethnopolitical Conflict in the Caucasus, London - New York : Routledge, 2000.

Gilligan 2009 : Emma Gilligan, Terror in Chechnya: Russia and the Tragedy of Civilians in War (Human Rights and Crimes Against Humanity), Princeton : Princeton University Press, 2009.

Gnabé 2002 : Laure Gnabé, « Les Tchétchènes n'attendent rien de personne : trois questions à Jean-Hervé Bradol », Le Nouvel Observateur, 25.10.2002.

Izmaïlov 2003 : Viatcheslav Izmaïlov, « Information à sensation ou totale fabrication », Novaïa Gazeta, 15.12.2003 (extraits traduits en anglais et en français in Binet 2010).

Izmaïlov 2004 : Viatcheslav Izmaïlov, « Le financeur de Khattab », Novaïa Gazeta, 16.02.2004 (extraits traduits en anglais et en français in Binet 2010).

Le Huérou et Regamey 2012 : Anne Le Huérou et Amandine Regamey, « Massacres de civils en Tchétchénie », Encyclopédie en ligne des violences de masse, document en ligne, publié le 11.10.2012, consulté le 05.05.2014 <http://www.massviolence.org/Massacres-decivils-en-Tchetchenie>.

Le Parisien 1995 : «Les Russes empêchent l'évacuation des blessés », Le Parisien, 21.01.1995.

Mac Mahon 2000 : Colin Mac Mahon, « Chechnya in Clutches of Quicksand Conflict », Chicago Tribune, 21.12.2000.

MSF 1995 : «Le droit humanitaire bafoué en Tchétchénie : l'exemple de Chatoï », Rapport MSF, 16.06.1995 (in Binet 2010).

MSF 1996 : «La Tchétchénie, loin de la paix », Rapport MSF, avril 1996 (in Binet 2010). 
MSF 1999a : Courriel de l'adjoint à la responsable juridique aux responsables de MSF France, 03.11.1999 (in Binet 2010).

MSF 1999b : «MSF demande une nouvelle fois l'ouverture immédiate de la frontière géorgienne et l'accueil des réfugiés tchétchènes par des pays tiers ", Communiqué de presse de MSF, 23.12.1999 (in Binet 2010).

MSF 1999c : «La traque des civils », interviews réalisées auprès de réfugiés tchétchènes en Géorgie, décembre 1999 (in Binet 2010).

MSF 1999d : Discours de MSF à la remise du prix Nobel de la paix - The Nobel Prize acceptance Speeech, 07.12.1999, document en ligne, consulté le 05.05.2014<http:// www.doctorswithoutborders.org/article/nobel-prize-acceptance-speech>.

MSF 2000a : « Les pays membres du Conseil de l'Europe doivent reconnaître l'état de guerre en Tchétchénie », Lettre ouverte de MSF aux États membres du Conseil de l'Europe, 26.01.2000 (in Binet 2010).

MSF 2000b : « Déclaration de MSF Tchétchénie à la presse », Courriel du chef de mission de MSF, 01.03.2000 (in Binet 2010), document en ligne, consulté le 09.11.2014<http:// speakingout.msf.org/en/node/2685>.

MSF 2000c : Lettre de la chargée des relations avec l'ONU de MSF à Sergueï Lavrov, ambassadeur extraordinaire et plénipotentiaire, Mission permanente de la Fédération de Russie à l'ONU, 16.03.2000, document en ligne, consulté le 09.11.2014<http:// speakingout.msf.org/en/node/2689>.

MSF 2000d : «Réfugiés tchétchènes en Ingouchie, résultats d'une enquête de Médecins Sans Frontières », 15.08.2000 (in Binet 2010).

MSF 2001 : «Brainstorming comm », Courriel de la responsable de l'information de MSF en Fédération de Russie, 15.01.2001 (in Binet 2010).

MSF 2002a : "Texte de l'audition de Médecins Sans Frontières devant l'Assemblée parlementaire du Conseil de l'Europe », 22.01.2002 (in Binet 2010).

MSF 2002b : « Le conseil de l'Europe : de la coopération à la collaboration », Communiqué de presse MSF, 24.01.2002 (in Binet 2010).

MSF 2002c : « Tchétchénie-Ingouchie : non assistance à personnes en danger 》, Rapport de MSF, janvier - février 2002 (in Binet 2010).

MSF 2002d : Sitrep n02-03 de MSF Belgique, avril 2002 (in Binet 2010).

MSF 2002e: « Briefing de Médecins Sans Frontières devant la $58^{\text {ème }}$ session de la Commission des droits de l'homme de l'ONU », 02.04.2002 (in Binet 2010).

MSF 2002f : «Les recommandations cosmétiques du Parlement européen », Communiqué de presse MSF, 11.04.2002 (in Binet 2010).

MSF 2002g : «MSF condamne la relocalisation forcée de déplacés tchétchènes », Communiqué de presse de MSF, 09.07.2002 (in Binet 2010).

MSF 2002h : « Déclaration de MSF au sujet de la prise d'otage de Moscou », Communiqué de presse de MSF, Moscou, 25.10.2002 (in Binet 2010).

MSF 2003a : "Appel international pour la libération d'Arjan, volontaire de MSF, otage depuis 7 mois dans le Caucase, à l'occasion de son anniversaire - Les autorités russes n'assument pas leurs responsabilités », Communiqué de presse de MSF, 07.03.2003 (in Binet 2010).

MSF 2003b : Lettre des chefs de mission de MSF en Fédération de Russie à Muratz Ziazikov, Président d'Ingouchie, 12.03.2003 (in Binet 2010).

MSF 2003c « Sans l'ombre d'un choix : le retour forcé des Tchétchènes en Tchétchénie », Enquête de MSF, avril 2003 (in Binet 2010). 
MSF 2003d : «En Russie, MSF abandonne le traitement contre la tuberculose dans la région de Kémérovo », Communiqué de presse de MSF, 30.09.2003.

MSF 2004 : « Médecins Sans Frontières confirme l'implication de représentants des autorités russes et daguestanaises dans l'enlèvement d'Arjan Erkel », Déclaration du Président et de la Secrétaire générale du mouvement MSF, 16.03.2004.

New York Times 2001 : « Kidnappers in Chechnya Urged to Free Brooklyn Man », The New York Times, 13.01.2001.

Sokirianskaya 2014 : Ekaterina Sokirianskaya, « State and Violence in Chechnya (19971999) », in A. Le Huérou et al., Chechnya at War and Beyond, London \& New York : Routledge, 2014, pp. 93-117.

Talbi 2002 : Karim Talbi, « Regain d'activité des rebelles tchétchènes », Le Figaro, 21.08.2002.

Van Zwol 2003a : Coen Van Zwol, «L'identité du ravisseur d'Arjan Erkel est connue », NRC Handelsblad, 05.11.2003 (extraits traduits en anglais et en français in Binet 2010).

Van Zwol 2003b : Coen Van Zwol, " La mort comme gagne-pain », NRC Handelsblad, 16.11.2003 (extraits traduits en anglais et en français in Binet 2010).

Veldkamp et Van Zwol, 2003 : Fenneken Veldkamp et Coen Van Zwol, « Un jeune homme aux cheveux clairs vaut des millions au Dagestan », NRC Handelsblad, 05.04 .2003 (extraits traduits en anglais et en français in Binet 2010). 